\title{
Refined converses of Jensen's inequality for operators
}

\author{
Jadranka Mićićc ${ }^{*}$, Josip Pečarić ${ }^{2}$ and Jurica Perić ${ }^{3}$
}

\footnotetext{
"Correspondence: jmicic@fsb.hr

${ }^{1}$ Faculty of Mechanical Engineering and Naval Architecture, University of Zagreb, Ivana Lučića 5, Zagreb,

10000, Croatia

Full list of author information is

available at the end of the article
}

\begin{abstract}
In this paper converses of a generalized Jensen's inequality for a continuous field of self-adjoint operators, a unital field of positive linear mappings and real-valued continuous convex functions are studied. New refined converses are presented by using the Mond-Pečarić method improvement. Obtained results are applied to refine selected inequalities with power functions.
\end{abstract}

MSC: 47A63; 47A64

Keywords: Mond-Pečarić method; self-adjoint operator; positive linear mapping; converse of Jensen's operator inequality; convex function

\section{Introduction}

Let $T$ be a locally compact Hausdorff space and let $\mathcal{A}$ be a $C^{*}$-algebra of operators on some Hilbert space $H$. We say that a field $\left(x_{t}\right)_{t \in T}$ of operators in $\mathcal{A}$ is continuous if the function $t \mapsto x_{t}$ is norm continuous on $T$. If in addition $\mu$ is a Radon measure on $T$ and the function $t \mapsto\left\|x_{t}\right\|$ is integrable, then we can form the Bochner integral $\int_{T} x_{t} d \mu(t)$, which is the unique element in $\mathcal{A}$ such that

$$
\varphi\left(\int_{T} x_{t} d \mu(t)\right)=\int_{T} \varphi\left(x_{t}\right) d \mu(t)
$$

for every linear functional $\varphi$ in the norm dual $\mathcal{A}^{*}$.

Assume further that there is a field $\left(\phi_{t}\right)_{t \in T}$ of positive linear mappings $\phi_{t}: \mathcal{A} \rightarrow \mathcal{B}$ from $\mathcal{A}$ to another $\mathcal{C}^{*}$-algebra $\mathcal{B}$ of operators on a Hilbert space $K$. We recall that a linear mapping $\phi: \mathcal{A} \rightarrow \mathcal{B}$ is said to be positive if $\phi(x) \geq 0$ for all $x \geq 0$. We say that such a field $\left(\phi_{t}\right)_{t \in T}$ is continuous if the function $t \mapsto \phi_{t}(x)$ is continuous for every $x \in \mathcal{A}$. Let the $\mathcal{C}^{*}$-algebras include the identity operators and let the function $t \mapsto \phi_{t}\left(1_{H}\right)$ be integrable with $\int_{T} \phi_{t}\left(1_{H}\right) d \mu(t)=k 1_{K}$ for some positive scalar $k$. If $\int_{T} \phi_{t}\left(1_{H}\right) d \mu(t)=1_{K}$, we say that a field $\left(\phi_{t}\right)_{t \in T}$ is unital.

Let $B(H)$ be the $C^{*}$-algebra of all bounded linear operators on a Hilbert space $H$. We define bounds of a self-adjoint operator $x \in B(H)$ by

$$
m_{x}:=\inf _{\|\xi\|=1}\langle x \xi, \xi\rangle \quad \text { and } \quad M_{x}:=\sup _{\|\xi\|=1}\langle x \xi, \xi\rangle
$$

for $\xi \in H$. If $\operatorname{Sp}(x)$ denotes the spectrum of $x$, then $\operatorname{Sp}(x) \subseteq\left[m_{x}, M_{x}\right]$ 
For an operator $x \in B(H)$, we define the operator $|x|:=\left(x^{*} x\right)^{1 / 2}$. Obviously, if $x$ is selfadjoint, then $|x|=\left(x^{2}\right)^{1 / 2}$.

Jensen's inequality is one of the most important inequalities. It has many applications in mathematics and statistics and some other well-known inequalities are its special cases.

Let $f$ be an operator convex function defined on an interval $I$. Davis [1] proved the socalled Jensen operator inequality

$$
f(\phi(x)) \leq \phi(f(x))
$$

where $\phi: \mathcal{A} \rightarrow B(K)$ is a unital completely positive linear mapping from a $C^{*}$-algebra $\mathcal{A}$ to linear operators on a Hilbert space $K$, and $x$ is a self-adjoint element in $\mathcal{A}$ with spectrum in $I$. Subsequently, Choi [2] noted that it is enough to assume that $\phi$ is unital and positive.

Mond, Pečarić, Hansen, Pedersen et al. in [3-6] studied another generalization of (2) for operator convex functions. Moreover, Hansen et al. [7] presented a general formulation of Jensen's operator inequality for a bounded continuous field of self-adjoint operators and a unital field of positive linear mappings:

$$
f\left(\int_{T} \phi_{t}\left(x_{t}\right) d \mu(t)\right) \leq \int_{T} \phi_{t}\left(f\left(x_{t}\right)\right) d \mu(t)
$$

where $f$ is an operator convex function.

There is an extensive literature devoted to Jensen's inequality concerning different refinements and extensive results, e.g., see [8-20]. Mićić et al. [21] proved that the discrete version of (3) stands without operator convexity of $f$ under a condition on the spectra of operators. Recently, Mićić et al. [22] presented a discrete version of refined Jensen's inequality for real-valued continuous convex functions. A continuous version is given below.

Theorem 1 Let $\left(x_{t}\right)_{t \in T}$ be a bounded continuous field of self-adjoint elements in a unital $C^{*}$-algebra $\mathcal{A}$ defined on a locally compact Hausdorff space $T$ equipped with a bounded Radon measure $\mu$. Let $m_{t}$ and $M_{t}, m_{t} \leq M_{t}$, be the bounds of $x_{t}, t \in T$. Let $\left(\phi_{t}\right)_{t \in T}$ be a unital field of positive linear mappings $\phi_{t}: \mathcal{A} \rightarrow \mathcal{B}$ from $\mathcal{A}$ to another unital $C^{*}$-algebra $\mathcal{B}$. Let

$$
\left(m_{x}, M_{x}\right) \cap\left[m_{t}, M_{t}\right]=\emptyset, \quad t \in T, \text { and } a<b,
$$

where $m_{x}$ and $M_{x}, m_{x} \leq M_{x}$, are the bounds of the operator $x=\int_{T} \phi_{t}\left(x_{t}\right) d \mu(t)$ and

$$
a=\sup \left\{M_{t}: M_{t} \leq m_{x}, t \in T\right\}, \quad b=\inf \left\{m_{t}: m_{t} \geq M_{x}, t \in T\right\} .
$$

If $: I \rightarrow \mathbb{R}$ is a continuous convex (resp. concave) function provided that the interval I contains all $m_{t}, M_{t}$, then

$$
f\left(\int_{T} \phi_{t}\left(x_{t}\right) d \mu(t)\right) \leq \int_{T} \phi_{t}\left(f\left(x_{t}\right)\right) d \mu(t)-\delta_{f} \bar{x} \leq \int_{T} \phi_{t}\left(f\left(x_{t}\right)\right) d \mu(t)
$$

(resp.

$$
\left.f\left(\int_{T} \phi_{t}\left(x_{t}\right) d \mu(t)\right) \geq \int_{T} \phi_{t}\left(f\left(x_{t}\right)\right) d \mu(t)-\delta_{f} \bar{x} \geq \int_{T} \phi_{t}\left(f\left(x_{t}\right)\right) d \mu(t)\right)
$$


holds, where

$$
\begin{aligned}
& \delta_{f} \equiv \delta_{f}(\bar{m}, \bar{M})=f(\bar{m})+f(\bar{M})-2 f\left(\frac{\bar{m}+\bar{M}}{2}\right) \\
& \left(\text { resp. } \delta_{f} \equiv \delta_{f}(\bar{m}, \bar{M})=2 f\left(\frac{\bar{m}+\bar{M}}{2}\right)-f(\bar{m})-f(\bar{M})\right), \\
& \bar{x} \equiv \bar{x}_{x}(\bar{m}, \bar{M})=\frac{1}{2} 1_{K}-\frac{1}{\bar{M}-\bar{m}}\left|x-\frac{\bar{m}+\bar{M}}{2} 1_{K}\right|
\end{aligned}
$$

and $\bar{m} \in\left[a, m_{x}\right], \bar{M} \in\left[M_{x}, b\right], \bar{m}<\bar{M}$, are arbitrary numbers.

The proof is similar to [22, Theorem 3] and we omit it.

On the other hand, Mond, Pečarić, Furuta et al. in [6, 23-27] investigated converses of Jensen's inequality. For presenting these results, we introduce some abbreviations. Let $f:[m, M] \rightarrow \mathbb{R}, m<M$. Then a linear function through $(m, f(m))$ and $(M, f(M))$ has the form $h(z)=k_{f} z+l_{f}$, where

$$
k_{f}:=\frac{f(M)-f(m)}{M-m} \quad \text { and } \quad l_{f}:=\frac{M f(m)-m f(M)}{M-m} .
$$

Using the Mond-Pečarić method, in [27] the following generalized converse of Jensen's operator inequality (2) is presented

$$
F[\phi(f(A)), g(\phi(A))] \leq \max _{m \leq z \leq M} F\left[k_{f} z+l_{f}, g(z)\right] 1_{\tilde{n}},
$$

for a convex function $f$ defined on an interval $[m, M], m<M$, where $g$ is a real-valued continuous function on $[m, M], F(u, v)$ is a real-valued function defined on $U \times V$, operator monotone in $u, U \supset f[m, M], V \supset g[m, M], \phi: H_{n} \rightarrow H_{\tilde{n}}$ is a unital positive linear mapping and $A$ is a self-adjoint operator with spectrum contained in $[m, M]$.

A continuous version of (6) and in the case of $\int_{T} \phi_{t}\left(1_{H}\right) d \mu(t)=k 1_{K}$ for some positive scalar $k$, is presented in [28]. Recently, Mićić et al. [29] obtained better bound than the one given in (6) as follows.

Theorem 2 [29, Theorem 2.1] Let $\left(x_{t}\right)_{t \in T}$ be a bounded continuous field of self-adjoint elements in a unital $C^{*}$-algebra $\mathcal{A}$ with the spectra in $[m, M], m<M$, defined on a locally compact Hausdorff space T equipped with a bounded Radon measure $\mu$, and let $\left(\phi_{t}\right)_{t \in T}$ be a unital field of positive linear maps $\phi_{t}: \mathcal{A} \rightarrow \mathcal{B}$ from $\mathcal{A}$ to another unital $C^{*}$-algebra $\mathcal{B}$. Let $m_{x}$ and $M_{x}, m_{x} \leq M_{x}$, be the bounds of the self-adjoint operator $x=\int_{T} \phi_{t}\left(x_{t}\right) d \mu(t)$ and $f:[m, M] \rightarrow \mathbb{R}, g:\left[m_{x}, M_{x}\right] \rightarrow \mathbb{R}, F: U \times V \rightarrow \mathbb{R}$, where $f([m, M]) \subseteq U, g\left(\left[m_{x}, M_{x}\right]\right) \subseteq V$ and $F$ is bounded.

Iff is convex and $F$ is an operator monotone in the first variable, then

$$
F\left[\int_{T} \phi_{t}\left(f\left(x_{t}\right)\right) d \mu(t), g\left(\int_{T} \phi_{t}\left(x_{t}\right) d \mu(t)\right)\right] \leq C_{1} 1_{K} \leq C 1_{K},
$$


where constants $C_{1} \equiv C_{1}\left(F, f, g, m, M, m_{x}, M_{x}\right)$ and $C \equiv C(F, f, g, m, M)$ are

$$
\begin{aligned}
C_{1} & =\sup _{m_{x} \leq z \leq M_{x}} F\left[k_{f} z+l_{f}, g(z)\right] \\
& =\sup _{\frac{M-M_{x}}{M-m} \leq p \leq \frac{M-m_{x}}{M-m}} F[p f(m)+(1-p) f(M), g(p m+(1-p) M)], \\
C & =\sup _{m \leq z \leq M} F\left[k_{f} z+l_{f}, g(z)\right] \\
& =\sup _{0 \leq p \leq 1} F[p f(m)+(1-p) f(M), g(p m+(1-p) M)] .
\end{aligned}
$$

If $f$ is concave, then reverse inequalities are valid in (7) with inf instead of sup in bounds $C_{1}$ and $C$.

In this paper, we present refined converses of Jensen's operator inequality. Applying these results, we further refine selected inequalities with power functions.

\section{Main results}

In the following we assume that $\left(x_{t}\right)_{t \in T}$ is a bounded continuous field of self-adjoint elements in a unital $C^{*}$-algebra $\mathcal{A}$ with the spectra in $[m, M], m<M$, defined on a locally compact Hausdorff space $T$ equipped with a bounded Radon measure $\mu$ and that $\left(\phi_{t}\right)_{t \in T}$ is a unital field of positive linear mappings $\phi_{t}: \mathcal{A} \rightarrow \mathcal{B}$ between $C^{*}$-algebras.

For convenience, we introduce abbreviations $\tilde{x}$ and $\delta_{f}$ as follows:

$$
\widetilde{x} \equiv \tilde{x}_{x_{t}, \phi_{t}}(m, M):=\frac{1}{2} 1_{K}-\frac{1}{M-m} \int_{T} \phi_{t}\left(\left|x_{t}-\frac{m+M}{2} 1_{H}\right|\right) d \mu(t)
$$

where $m, M, m<M$, are some scalars such that the spectra of $x_{t}, t \in T$, are in $[m, M]$;

$$
\delta_{f} \equiv \delta_{f}(m, M):=f(m)+f(M)-2 f\left(\frac{m+M}{2}\right)
$$

where $f:[m, M] \rightarrow \mathbb{R}$ is a continuous function.

Obviously, $m 1_{H} \leq x_{t} \leq M 1_{H}$ implies $-\frac{M-m}{2} 1_{H} \leq x_{t}-\frac{m+M}{2} 1_{H} \leq \frac{M-m}{2} 1_{H}$ for $t \in T$ and $\int_{T} \phi_{t}\left(\left|x_{t}-\frac{m+M}{2} 1_{H}\right|\right) d \mu(t) \leq \frac{M-m}{2} \int_{T} \phi_{t}\left(1_{H}\right) d \mu(t)=\frac{M-m}{2} 1_{K}$. It follows $\widetilde{x} \geq 0$. Also, if $f$ is convex (resp. concave), then $\delta_{f} \geq 0$ (resp. $\delta_{f} \leq 0$ ).

To prove our main result related to converse Jensen's inequality, we need the following lemma.

Lemma 3 Let $f$ be a convex function on an interval $I, m, M \in I$ and $p_{1}, p_{2} \in[0,1]$ such that $p_{1}+p_{2}=1$. Then

$$
\begin{aligned}
& \min \left\{p_{1}, p_{2}\right\}\left[f(m)+f(M)-2 f\left(\frac{m+M}{2}\right)\right] \\
& \quad \leq p_{1} f(m)+p_{2} f(M)-f\left(p_{1} m+p_{2} M\right) \\
& \quad \leq \max \left\{p_{1}, p_{2}\right\}\left[f(m)+f(M)-2 f\left(\frac{m+M}{2}\right)\right] .
\end{aligned}
$$


Proof These results follow from [30, Theorem 1, p.717] for $n=2$. For the reader's convenience, we give an elementary proof of (10).

Let $a_{i} \leq b_{i}, i=1,2$, be positive real numbers such that $A=a_{1}+a_{2}<B=b_{1}+b_{2}$. Using Jensen's inequality and its reverse, we get

$$
\begin{aligned}
B f & \left(\frac{b_{1} m+b_{2} M}{B}\right)-A f\left(\frac{a_{1} m+a_{2} M}{A}\right) \\
& \leq(B-A) f\left(\frac{\left(b_{1}-a_{1}\right) m+\left(b_{2}-a_{2}\right) M}{B-A}\right) \\
& \leq\left(b_{1}-a_{1}\right) f(m)+\left(b_{2}-a_{2}\right) f(M) \\
& =b_{1} f(m)+b_{2} f_{2}(M)-\left(a_{1} f(m)+a_{2} f_{2}(M)\right) .
\end{aligned}
$$

Suppose that $0<p_{1}<p_{2}<1, p_{1}+p_{2}=1$. Replacing $a_{1}$ and $a_{2}$ by $p_{1}$ and $p_{2}$, respectively, and putting $b_{1}=b_{2}=p_{2}, A=1$ and $B=2 p_{2}$ in (11), we get

$$
2 p_{2} f\left(\frac{m+M}{2}\right)-f\left(p_{1} f(m)+p_{2} f(M)\right) \leq p_{2} f(m)+p_{2} f_{2}(M)-\left(p_{1} f(m)+p_{2} f_{2}(M)\right),
$$

which gives the right-hand side of (10). Similarly, replacing $b_{1}$ and $b_{2}$ by $p_{1}$ and $p_{2}$, respectively, and putting $a_{1}=a_{2}=p_{1}, A=2 p_{1}$ and $B=1$ in (11), we obtain the left-hand side of (10).

If $p_{1}=0, p_{2}=1$ or $p_{1}=1, p_{2}=0$, then inequality (10) holds, since $f$ is convex. If $p_{1}=p_{2}=$ $1 / 2$, then we have an equality in (10).

The main result of an improvement of the Mond-Pečarić method follows.

Lemma 4 Let $\left(x_{t}\right)_{t \in T},\left(\phi_{t}\right)_{t \in T}, m$ and $M$ be as above. Then

$$
\int_{T} \phi_{t}\left(f\left(x_{t}\right)\right) d \mu(t) \leq k_{f} \int_{T} \phi_{t}\left(x_{t}\right) d \mu(t)+l_{f} 1_{K}-\delta_{f} \tilde{x} \leq k_{f} \int_{T} \phi_{t}\left(x_{t}\right) d \mu(t)+l_{f} 1_{K}
$$

for every continuous convex function $f:[m, M] \rightarrow \mathbb{R}$, where $\tilde{x}$ and $\delta_{f}$ are defined by (8) and (9), respectively.

Iff is concave, then the reverse inequality is valid in (12).

Proof We prove only the convex case. By using (10) we get

$$
f\left(p_{1} m+p_{2} M\right) \leq p_{1} f(m)+p_{2} f(M)-\min \left\{p_{1}, p_{2}\right\}\left[f(m)+f(M)-2 f\left(\frac{m+M}{2}\right)\right]
$$

for every $p_{1}, p_{2} \in[0,1]$ such that $p_{1}+p_{2}=1$. Let functions $p_{1}, p_{2}:[m, M] \rightarrow[0,1]$ be defined by

$$
p_{1}(z)=\frac{M-z}{M-m}, \quad p_{2}(z)=\frac{z-m}{M-m} .
$$

Then, for any $z \in[m, M]$, we can write

$$
f(z)=f\left(\frac{M-z}{M-m} m+\frac{z-m}{M-m} M\right)=f\left(p_{1}(z) m+p_{2}(z) M\right) .
$$


By using (13) we get

$$
f(z) \leq \frac{M-z}{M-m} f(m)+\frac{z-m}{M-m} f(M)-\tilde{z}\left[f(m)+f(M)-2 f\left(\frac{m+M}{2}\right)\right]
$$

where

$$
\tilde{z}=\frac{1}{2}-\frac{1}{M-m}\left|z-\frac{m+M}{2}\right|
$$

since

$$
\min \left\{\frac{M-z}{M-m}, \frac{z-m}{M-m}\right\}=\frac{1}{2}-\frac{1}{M-m}\left|z-\frac{m+M}{2}\right|
$$

Now since $\operatorname{Sp}\left(x_{t}\right) \subseteq[m, M]$, by utilizing the functional calculus to (14), we obtain

$$
f\left(x_{t}\right) \leq \frac{M-x_{t}}{M-m} f(m)+\frac{x_{t}-m}{M-m} f(M)-\tilde{x}_{t}\left[f(m)+f(M)-2 f\left(\frac{m+M}{2}\right)\right],
$$

where

$$
\tilde{x}_{t}=\frac{1}{2} 1_{H}-\frac{1}{M-m}\left|x_{t}-\frac{m+M}{2} 1_{H}\right|
$$

Applying a positive linear mapping $\phi_{t}$, integrating and using $\int_{T} \phi_{t}\left(1_{H}\right) d \mu(t)=1_{K}$, we get the first inequality in (12) since

$$
\tilde{x}=\int_{T} \phi_{t}\left(\tilde{x}_{t}\right) d \mu(t)=\frac{1}{2} 1_{K}-\frac{1}{M-m} \int_{T} \phi_{t}\left(\left|x_{t}-\frac{m+M}{2} 1_{H}\right|\right) d \mu(t) .
$$

By using that $\delta_{f} \tilde{x} \geq 0$, the second inequality in (12) holds.

We can use Lemma 4 to obtain refinements of some other inequalities mentioned in the introduction. First, we present a refinement of Theorem 2.

Theorem 5 Let $m_{x}$ and $M_{x}, m_{x} \leq M_{x}$, be the bounds of the operator $x=\int_{T} \phi_{t}\left(x_{t}\right) d \mu(t)$ and let $m_{\tilde{x}}$ be the lower bound of the operator $\tilde{x}$. Let $f:[m, M] \rightarrow \mathbb{R}, g:\left[m_{x}, M_{x}\right] \rightarrow \mathbb{R}$, $F: U \times V \rightarrow \mathbb{R}$, where $f([m, M]) \subseteq U, g\left(\left[m_{x}, M_{x}\right]\right) \subseteq V$ and $F$ is bounded.

Iff is convex and $F$ is operator monotone in the first variable, then

$$
\begin{aligned}
& F\left[\int_{T} \phi_{t}\left(f\left(x_{t}\right)\right) d \mu(t), g\left(\int_{T} \phi_{t}\left(x_{t}\right) d \mu(t)\right)\right] \\
& \quad \leq F\left[k_{f} x+l_{f}-\delta_{f} \tilde{x}, g\left(\int_{T} \phi_{t}\left(x_{t}\right) d \mu(t)\right)\right] \\
& \quad \leq \sup _{m_{x} \leq z \leq M_{x}} F\left[k_{f} z+l_{f}-\delta_{f} m_{\widetilde{x}}, g(z)\right] 1_{K} \leq \sup _{m_{x} \leq z \leq M_{x}} F\left[k_{f} z+l_{f}, g(z)\right] 1_{K} .
\end{aligned}
$$

Iff is concave, then the reverse inequality is valid in (15) with inf instead of sup. 
Proof We prove only the convex case. Then $\delta_{f} \geq 0$ implies $0 \leq \delta_{f} m_{\widetilde{x}} 1_{K} \leq \delta_{f} \widetilde{x}$. By using (12) it follows that

$$
\int_{T} \phi_{t}\left(f\left(x_{t}\right)\right) d \mu(t) \leq k_{f} x+l_{f}-\delta_{f} \tilde{x} \leq k_{f} x+l_{f}-\delta_{f} m_{\tilde{x}} 1_{K} \leq k_{f} x+l_{f} .
$$

Using operator monotonicity of $F(\cdot, v)$ in the first variable, we obtain (15).

\section{Difference-type converse inequalities}

By using Jensen's operator inequality, we obtain that

$$
\alpha g\left(\int_{T} \phi_{t}\left(x_{t}\right) d \mu(t)\right) \leq \int_{T} \phi_{t}\left(f\left(x_{t}\right)\right) d \mu(t)
$$

holds for every operator convex function $f$ on $[m, M]$, every function $g$ and real number $\alpha$ such that $\alpha g \leq f$ on $[m, M]$. Now, applying Theorem 5 to the function $F(u, v)=u-\alpha v$, $\alpha \in \mathbb{R}$, we obtain the following converse of (16). It is also a refinement of [29, Theorem 3.1].

Theorem 6 Let $m_{x}$ and $M_{x}, m_{x} \leq M_{x}$, be the bounds of the operator $x=\int_{T} \phi_{t}\left(x_{t}\right) d \mu(t)$ and $f:[m, M] \rightarrow \mathbb{R}, g:\left[m_{x}, M_{x}\right] \rightarrow \mathbb{R}$ be continuous functions.

Iff is convex and $\alpha \in \mathbb{R}$, then

$$
\int_{T} \phi_{t}\left(f\left(x_{t}\right)\right) d \mu(t)-\alpha g\left(\int_{T} \phi_{t}\left(x_{t}\right) d \mu(t)\right) \leq \max _{m_{x} \leq z \leq M_{x}}\left\{k_{f} z+l_{f}-\alpha g(z)\right\} 1_{K}-\delta_{f} \tilde{x} .
$$

Iff is concave, then the reverse inequality is valid in (17) with min instead of max.

Remark 1 (1) Obviously,

$$
\begin{aligned}
& \int_{T} \phi_{t}\left(f\left(x_{t}\right)\right) d \mu(t)-\alpha g\left(\int_{T} \phi_{t}\left(x_{t}\right) d \mu(t)\right) \\
& \quad \leq \max _{m_{x} \leq z \leq M_{x}}\left\{k_{f} z+l_{f}-\alpha g(z)\right\} 1_{K}-\delta_{f} \tilde{y} \leq \max _{m_{x} \leq z \leq M_{x}}\left\{k_{f} z+l_{f}-\alpha g(z)\right\} 1_{K}
\end{aligned}
$$

for every convex function $f$, every $\alpha \in \mathbb{R}$, and $m_{\tilde{x}} 1_{K} \leq \tilde{y} \leq \tilde{x}$, where $m_{\widetilde{x}}$ is the lower bound of $\tilde{x}$.

(2) According to [29, Corollary 3.2], we can determine the constant in the RHS of (17).

(i) Let $f$ be convex. We can determine the value $C_{\alpha}$ in

$$
\int_{T} \phi_{t}\left(f\left(x_{t}\right)\right) d \mu(t)-\alpha g\left(\int_{T} \phi_{t}\left(x_{t}\right) d \mu(t)\right) \leq C_{\alpha} 1_{K}-\delta_{f} \tilde{x}
$$

as follows:

- if $\alpha \leq 0, g$ is convex or $\alpha \geq 0, g$ is concave, then

$$
C_{\alpha}=\max \left\{k_{f} m_{x}+l_{f}-\alpha g\left(m_{x}\right), k_{f} M_{x}+l_{f}-\alpha g\left(M_{x}\right)\right\}
$$


- if $\alpha \leq 0, g$ is concave or $\alpha \geq 0, g$ is convex, then

$$
C_{\alpha}= \begin{cases}k_{f} m_{x}+l_{f}-\alpha g\left(m_{x}\right) & \text { if } \alpha g_{-}^{\prime}(z) \geq k_{f} \text { for every } z \in\left(m_{x}, M_{x}\right), \\ k_{f} z_{0}+l_{f}-\alpha g\left(z_{0}\right) & \text { if } \alpha g_{-}^{\prime}\left(z_{0}\right) \leq k_{f} \leq \alpha g_{+}^{\prime}\left(z_{0}\right) \\ & \text { for some } z_{0} \in\left(m_{x}, M_{x}\right), \\ k_{f} M_{x}+l_{f}-\alpha g\left(M_{x}\right) & \text { if } \alpha g_{+}^{\prime}(z) \leq k_{f} \text { for every } z \in\left(m_{x}, M_{x}\right) .\end{cases}
$$

(ii) Let $f$ be concave. We can determine the value $c_{\alpha}$ in

$$
c_{\alpha} 1_{K}-\delta_{f} \tilde{x} \leq \int_{T} \phi_{t}\left(f\left(x_{t}\right)\right) d \mu(t)-\alpha g\left(\int_{T} \phi_{t}\left(x_{t}\right) d \mu(t)\right)
$$

as follows:

- if $\alpha \leq 0, g$ is convex or $\alpha \geq 0, g$ is concave, then $c_{\alpha}$ is equal to the right-hand side in (19) with reverse inequality signs;

- if $\alpha \leq 0, g$ is concave or $\alpha \geq 0, g$ is convex, then $c_{\alpha}$ is equal to the right-hand side in (18) with min instead of max.

Theorem 6 and Remark 1(2) applied to functions $f(z)=z^{p}$ and $g(z)=z^{q}$ give the following corollary, which is a refinement of [29, Corollary 3.3].

Corollary 7 Let $\left(x_{t}\right)_{t \in T}$ be a field of strictly positive operators, let $m_{x}$ and $M_{x}, m_{x} \leq M_{x}$, be the bounds of the operator $x=\int_{T} \phi_{t}\left(x_{t}\right) d \mu(t)$. Let $\widetilde{x}$ be defined by (8).

(i) Let $p \in(-\infty, 0] \cup[1, \infty)$. Then

$$
\int_{T} \phi_{t}\left(x_{t}^{p}\right) d \mu(t)-\alpha\left(\int_{T} \phi_{t}\left(x_{t}\right) d \mu(t)\right)^{q} \leq C_{\alpha}^{\star} 1_{K}-\left(m^{p}+M^{p}-2^{1-p}(m+M)^{p}\right) \widetilde{x}
$$

where the constant $C_{\alpha}^{\star}$ is determined as follows:

- if $\alpha \leq 0, q \in(-\infty, 0] \cup[1, \infty)$ or $\alpha \geq 0, q \in(0,1)$, then

$$
C_{\alpha}^{\star}=\max \left\{k_{t^{p}} m_{x}+l_{t^{p}}-\alpha m_{x}^{q}, k_{t^{p}} M_{x}+l_{t^{p}}-\alpha M_{x}^{q}\right\}
$$

- if $\alpha \leq 0, q \in(0,1)$ or $\alpha \geq 0, q \in(-\infty, 0] \cup[1, \infty)$, then

$$
C_{\alpha}^{\star}= \begin{cases}k_{t p} m_{x}+l_{t^{p}}-\alpha m_{x}^{q} & \text { if }\left(\alpha q / k_{t^{p}}\right)^{1 /(1-q)} \leq m_{x}, \\ l_{t^{p}}+\alpha(q-1)\left(\alpha q / k_{t^{p}}\right)^{q /(1-q)} & \text { if } m_{x} \leq\left(\alpha q / k_{t}\right)^{1 /(1-q)} \leq M_{x}, \\ k_{t^{p}} M_{x}+l_{t^{p}}-\alpha M_{x}^{q} & \text { if }\left(\alpha q / k_{t^{p}}\right)^{1 /(1-q)} \geq M_{x},\end{cases}
$$

where $k_{t^{p}}:=\left(M^{p}-m^{p}\right) /(M-m)$ and $l_{t^{p}}:=\left(M m^{p}-m M^{p}\right) /(M-m)$.

(ii) Let $p \in(0,1)$. Then

$$
c_{\alpha}^{\star} 1_{K}+\left(2^{1-p}(m+M)^{p}-m^{p}-M^{p}\right) \widetilde{x} \leq \int_{T} \phi_{t}\left(x_{t}^{p}\right) d \mu(t)-\alpha\left(\int_{T} \phi_{t}\left(x_{t}\right) d \mu(t)\right)^{q},
$$

where the constant $c_{\alpha}^{\star}$ is determined as follows: 
- if $\alpha \leq 0, q \in(-\infty, 0] \cup[1, \infty)$ or $\alpha \geq 0, q \in(0,1)$, then $c_{\alpha}^{\star}$ is equal to the right-hand side in (21):

- if $\alpha \leq 0, q \in(0,1)$ or $\alpha \geq 0, q \in(-\infty, 0] \cup[1, \infty)$, then $c_{\alpha}^{\star}$ is equal to the right-hand side in (20) with min instead of max.

Using Theorem 6 and Remark 1 for $g \equiv f$ and $\alpha=1$ and utilizing elementary calculations, we obtain the following converse of Jensen's inequality.

Theorem 8 Let $m_{x}$ and $M_{x}, m_{x} \leq M_{x}$, be the bounds of the operator $x=\int_{T} \phi_{t}\left(x_{t}\right) d \mu(t)$ and let $f:[m, M] \rightarrow \mathbb{R}$ be a continuous function.

Iff is convex, then

$$
0 \leq \int_{T} \phi_{t}\left(f\left(x_{t}\right)\right) d \mu(t)-f\left(\int_{T} \phi_{t}\left(x_{t}\right) d \mu(t)\right) \leq \bar{C} 1_{K}-\delta_{f} \tilde{x}
$$

where $\tilde{x}$ and $\delta_{f}$ are defined by (8) and (9), respectively, and

$$
\bar{C}=\max _{m_{x} \leq z \leq M_{x}}\left\{k_{f} z+l_{f}-f(z)\right\}
$$

Furthermore, iff is strictly convex differentiable, then the bound $\bar{C} 1_{K}-\delta_{f} \tilde{x}$ satisfies the following condition:

$$
0 \leq \bar{C} 1_{K}-\delta_{f} \tilde{x} \leq\left\{f(M)-f(m)-f^{\prime}(m)(M-m)-\delta_{f} m_{\tilde{x}}\right\} 1_{K},
$$

where $m_{\tilde{x}}$ is the lower bound of the operator $\tilde{x}$. We can determine the value $\bar{C}$ in (23) as follows:

$$
\bar{C}=k_{f} z_{0}+l_{f}-f\left(z_{0}\right),
$$

where

$$
z_{0}= \begin{cases}m_{x} & \text { if } f^{\prime}\left(m_{x}\right) \geq k_{f}, \\ f^{\prime-1}\left(k_{f}\right) & \text { if } f^{\prime}\left(m_{x}\right) \leq k_{f} \leq f^{\prime}\left(M_{x}\right), \\ M_{x} & \text { if } f^{\prime}\left(M_{x}\right) \leq k_{f} .\end{cases}
$$

In the dual case, when $f$ is concave, the reverse inequality is valid in (22) with min instead of max in (23). Furthermore, iff is strictly concave differentiable, then the bound $\bar{C} 1_{K}-\delta_{f} \tilde{x}$ satisfies the following condition:

$$
\left\{f(M)-f(m)-f^{\prime}(m)(M-m)-\delta_{f} m_{\tilde{x}}\right\} 1_{K} \leq \bar{C} 1_{K}-\delta_{f} \tilde{x} \leq 0 .
$$

We can determine the value $\bar{C}$ in (24) with $z_{0}$, which equals the right-hand side in (25) with reverse inequality signs.

Example 1 We give examples for the matrix cases and $T=\{1,2\}$. We put $f(t)=t^{4}$, which is convex, but not operator convex. Also, we define mappings $\Phi_{1}, \Phi_{2}: M_{3}(\mathbb{C}) \rightarrow M_{2}(\mathbb{C})$ by $\Phi_{1}\left(\left(a_{i j}\right)_{1 \leq i, j \leq 3}\right)=\frac{1}{2}\left(a_{i j}\right)_{1 \leq i, j \leq 2}, \Phi_{2}=\Phi_{1}$ and measures by $\mu(\{1\})=\mu(\{2\})=1$. 


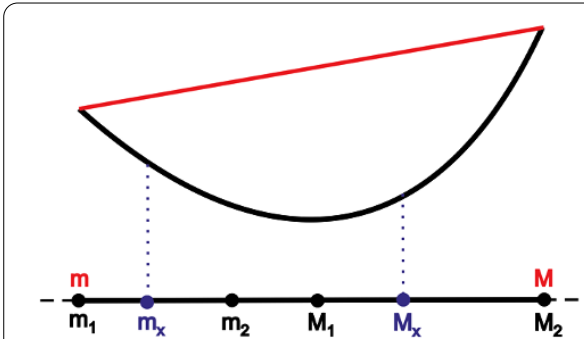

a)

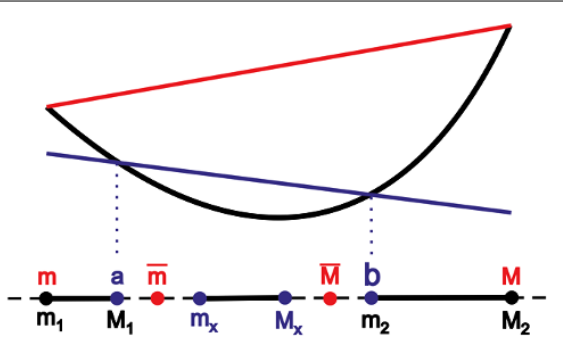

b)

Figure 1 Refinement for two operators and a convex function $f$.

(I) First, we observe an example without the spectra condition (see Figure 1(a)). Then we obtain a refined inequality as in (22), but do not have refined Jensen's inequality.

$$
\text { If } X_{1}=2\left(\begin{array}{lll}
1 & 0 & 1 \\
0 & 0 & 1 \\
1 & 1 & 1
\end{array}\right) \quad \text { and } \quad X_{2}=2\left(\begin{array}{lll}
1 & 0 & 0 \\
0 & 0 & 0 \\
0 & 0 & 0
\end{array}\right) \text {, then } X=2\left(\begin{array}{ll}
1 & 0 \\
0 & 0
\end{array}\right)
$$

and $m_{1}=-1.604, M_{1}=4.494, m_{2}=0, M_{2}=2, m=-1.604, M=4.494$ (rounded to three decimal places). We have

$$
\left(\Phi_{1}\left(X_{1}\right)+\Phi_{2}\left(X_{2}\right)\right)^{4}=\left(\begin{array}{cc}
16 & 0 \\
0 & 0
\end{array}\right) \supsetneqq\left(\begin{array}{cc}
80 & 40 \\
40 & 24
\end{array}\right)=\Phi_{1}\left(X_{1}^{4}\right)+\Phi_{2}\left(X_{2}^{4}\right)
$$

and

$$
\begin{aligned}
\Phi_{1}\left(X_{1}^{4}\right)+\Phi_{2}\left(X_{2}^{4}\right) & =\left(\begin{array}{ll}
80 & 40 \\
40 & 24
\end{array}\right) \\
<\Phi_{1}\left(X_{1}^{4}\right)+\Phi_{2}\left(X_{2}^{4}\right)+\bar{C} I_{2}-\delta_{f} \tilde{X} & =\left(\begin{array}{cc}
111.742 & 39.327 \\
39.327 & 142.858
\end{array}\right) \\
<\left(\Phi_{1}\left(X_{1}\right)+\Phi_{2}\left(X_{2}\right)\right)^{4}+\bar{C} I_{2} & =\left(\begin{array}{cc}
243.758 & 0 \\
0 & 227.758
\end{array}\right),
\end{aligned}
$$

since $\bar{C}=227.758, \delta_{f}=405.762, \widetilde{X}=\left(\begin{array}{cc}0.325 & -0.097 \\ -0.097 & 0.2092\end{array}\right)$.

(II) Next, we observe an example with the spectra condition (see Figure 1(b)). Then we obtain a series of inequalities involving refined Jensen's inequality and its converses.

$$
\text { If } X_{1}=\left(\begin{array}{ccc}
-4 & 1 & 1 \\
1 & -2 & -1 \\
1 & -1 & -1
\end{array}\right) \quad \text { and } \quad X_{2}=\left(\begin{array}{ccc}
5 & -1 & -1 \\
-1 & 2 & 1 \\
-1 & 1 & 3
\end{array}\right) \text {, then } X=\frac{1}{2}\left(\begin{array}{ll}
1 & 0 \\
0 & 0
\end{array}\right)
$$

and $m_{1}=-4.866, M_{1}=-0.345, m_{2}=1.345, M_{2}=5.866, m=-4.866, M=5.866, a=$ $-0.345, b=1.345$ and we put $\bar{m}=a, \bar{M}=b$ (rounded to three decimal places). We 
have

$$
\begin{array}{ll}
\left(\Phi_{1}\left(X_{1}\right)+\Phi_{2}\left(X_{2}\right)\right)^{4} & =\left(\begin{array}{cc}
0.0625 & 0 \\
0 & 0
\end{array}\right) \\
<\Phi_{1}\left(X_{1}^{4}\right)+\Phi_{2}\left(X_{2}^{4}\right)-\delta_{f}(a, b) \bar{X} & =\left(\begin{array}{cc}
639.921 & -255 \\
-255 & 117.856
\end{array}\right) \\
<\Phi_{1}\left(X_{1}^{4}\right)+\Phi_{2}\left(X_{2}^{4}\right) & =\left(\begin{array}{cc}
641.5 & -255 \\
-255 & 118.5
\end{array}\right) \\
<\left(\Phi_{1}\left(X_{1}\right)+\Phi_{2}\left(X_{2}\right)\right)^{4}+\bar{C} I_{2}-\delta_{f}(m, M) \widetilde{X} & =\left(\begin{array}{cc}
731.649 & -162.575 \\
-162.575 & 325.15
\end{array}\right) \\
<\left(\Phi_{1}\left(X_{1}\right)+\Phi_{2}\left(X_{2}\right)\right)^{4}+\bar{C} I_{2} & =\left(\begin{array}{cc}
872.471 & 0 \\
0 & 872.409
\end{array}\right),
\end{array}
$$

since $\delta_{f}(a, b)=3.158, \bar{X}=\left(\begin{array}{cc}0.5 & 0 \\ 0 & 0.204\end{array}\right), \delta_{f}(m, M)=1744.82, \tilde{X}=\left(\begin{array}{cc}0.325 & -0.097 \\ -0.097 & 0.2092\end{array}\right)$ and $\bar{C}=$ 872.409 .

Applying Theorem 8 to $f(t)=t^{p}$, we obtain the following refinement of [29, Corollary 3.6].

Corollary 9 Let $\left(x_{t}\right)_{t \in T}$ be a field of strictly positive operators, let $m_{x}$ and $M_{x}, m_{x} \leq M_{x}$, be the bounds of the operator $x=\int_{T} \phi_{t}\left(x_{t}\right) d \mu(t)$. Let $\tilde{x}$ be defined by (8). Then

$$
\begin{aligned}
0 & \leq \int_{T} \phi_{t}\left(x_{t}^{p}\right) d \mu(t)-\left(\int_{T} \phi_{t}\left(x_{t}\right) d \mu(t)\right)^{p} \\
& \leq \bar{C}\left(m_{x}, M_{x}, m, M, p\right) 1_{K}-\left(m^{p}+M^{p}-2^{1-p}(m+M)^{p}\right) \widetilde{x} \\
& \leq \bar{C}\left(m_{x}, M_{x}, m, M, p\right) 1_{K} \leq C(m, M, p) 1_{K}
\end{aligned}
$$

for $p \notin(0,1)$, and

$$
\begin{aligned}
C(m, M, p) 1_{K} & \leq \bar{c}\left(m_{x}, M_{x}, m, M, p\right) 1_{K} \\
& \leq \bar{c}\left(m_{x}, M_{x}, m, M, p\right) 1_{K}+\left(2^{1-p}(m+M)^{p}-m^{p}-M^{p}\right) \widetilde{x} \\
& \leq \int_{T} \phi_{t}\left(x_{t}^{p}\right) d \mu(t)-\left(\int_{T} \phi_{t}\left(x_{t}\right) d \mu(t)\right)^{p} \leq 0
\end{aligned}
$$

for $p \in(0,1)$, where

$$
\bar{C}\left(m_{x}, M_{x}, m, M, p\right)= \begin{cases}k_{t^{p}} m_{x}+l_{t^{p}}-m_{x}^{p} & \text { if } p m_{x}^{p-1} \geq k_{t^{p}}, \\ C(m, M, p) & \text { if } p m_{x}^{p-1} \leq k_{t^{p}} \leq p M_{x}^{p-1} \\ k_{t^{p}} M_{x}+l_{t^{p}}-M_{x}^{p} & \text { if } p M_{x}^{p-1} \leq k_{t},\end{cases}
$$

and $\bar{c}\left(m_{x}, M_{x}, m, M, p\right)$ equals the right-hand side in (26) with reverse inequality signs. $C(m, M, p)$ is the known Kantorovich-type constant for difference (see, i.e., [6, §2.7]):

$$
C(m, M, p)=(p-1)\left(\frac{M^{p}-m^{p}}{p(M-m)}\right)^{1 /(p-1)}+\frac{M m^{p}-m M^{p}}{M-m} \text { for } p \in \mathbb{R} .
$$




\section{Ratio-type converse inequalities}

In [29, Theorem 4.1] the following ratio-type converse of (16) is given:

$$
\int_{T} \phi_{t}\left(f\left(x_{t}\right)\right) d \mu(t) \leq \max _{m_{x} \leq z \leq M_{x}}\left\{\frac{k_{f} z+l_{f}}{g(z)}\right\} g\left(\int_{T} \phi_{t}\left(x_{t}\right) d \mu(t)\right),
$$

where $f$ is convex and $g>0$. Applying Theorem 5 and Theorem 6, we obtain the following two refinements of (27).

Theorem 10 Let $m_{x}$ and $M_{x}, m_{x} \leq M_{x}$, be the bounds of the operator $x=\int_{T} \phi_{t}\left(x_{t}\right) d \mu(t)$ and let $f:[m, M] \rightarrow \mathbb{R}, g:\left[m_{x}, M_{x}\right] \rightarrow \mathbb{R}$ be continuous functions.

Iff is convex and $g>0$, then

$$
\int_{T} \phi_{t}\left(f\left(x_{t}\right)\right) d \mu(t) \leq \max _{m_{x} \leq z \leq M_{x}}\left\{\frac{k_{f} z+l_{f}}{g(z)}\right\} g\left(\int_{T} \phi_{t}\left(x_{t}\right) d \mu(t)\right)-\delta_{f} \widetilde{x}
$$

and

$$
\int_{T} \phi_{t}\left(f\left(x_{t}\right)\right) d \mu(t) \leq \max _{m_{x} \leq z \leq M_{x}}\left\{\frac{k_{f} z+l_{f}-\delta_{f} m_{\tilde{x}}}{g(z)}\right\} g\left(\int_{T} \phi_{t}\left(x_{t}\right) d \mu(t)\right),
$$

where $\tilde{x}$ and $\delta_{f}$ are defined by (8) and (9), respectively, and $m_{\tilde{x}}$ is the lower bound of the operator $\tilde{x}$. If $f$ is concave, then reverse inequalities are valid in (28) and (29) with min instead of max.

Proof We prove only the convex case. Let $\alpha_{1}=\max _{m_{x} \leq z \leq M_{x}}\left\{\frac{k_{f} z+l_{f}}{g(z)}\right\}$. Then there is $z_{0} \in$ $\left[m_{x}, M_{x}\right]$ such that $\alpha_{1}=\frac{k_{f} z_{0}+l_{f}}{g\left(z_{0}\right)}$ and $\frac{k_{f} z+l_{f}}{g(z)} \leq \alpha_{1}$ for all $z \in\left[m_{x}, M_{x}\right]$. It follows that $k_{f} z_{0}+l_{f}-$ $\alpha_{1} g\left(z_{0}\right)=0$ and $k_{f} z+l_{f}-\alpha_{1} g(z) \leq 0$ for all $z \in\left[m_{x}, M_{x}\right]$. So,

$$
\max _{m_{x} \leq z \leq M_{x}}\left\{k_{f} z+l_{f}-\alpha_{1} g(z)\right\}=0
$$

By using (17), we obtain (28). Inequality (29) follows directly from Theorem 5 by putting $F(u, v)=v^{-1 / 2} u v^{-1 / 2}$.

Remark 2 (1) Inequality (28) is a refinement of (27) since $\delta_{f} \tilde{x} \geq 0$. Also, (29) is a refinement of (27) since $m_{\widetilde{x}} \geq 0$ and $g>0$ implies

$$
\max _{m_{x} \leq z \leq M_{x}}\left\{\frac{k_{f} z+l_{f}-\delta_{f} m_{\tilde{x}}}{g(z)}\right\} \leq \max _{m_{x} \leq z \leq M_{x}}\left\{\frac{k_{f} z+l_{f}}{g(z)}\right\} .
$$

(2) Let the assumptions of Theorem 10 hold. Generally, there is no relation between the right-hand sides of inequalities (28) and (29) under the operator order (see Example 2). But, for example, if $g\left(\int_{T} \phi_{t}\left(x_{t}\right) d \mu(t)\right) \leq g\left(z_{0}\right) 1_{K}$, where $z_{0} \in\left[m_{x}, M_{x}\right]$ is the point where it achieves $\max _{m_{x} \leq z \leq M_{x}}\left\{\frac{k_{f} z+l_{f}}{g(z)}\right\}$, then the following order holds:

$$
\begin{aligned}
\int_{T} \phi_{t}\left(f\left(x_{t}\right)\right) d \mu(t) & \leq \max _{m_{x} \leq z \leq M_{x}}\left\{\frac{k_{f} z+l_{f}}{g(z)}\right\} g\left(\int_{T} \phi_{t}\left(x_{t}\right) d \mu(t)\right)-\delta_{f} \tilde{x} \\
& \leq \max _{m_{x} \leq z \leq M_{x}}\left\{\frac{k_{f} z+l_{f}-\delta_{f} m_{\tilde{x}}}{g(z)}\right\} g\left(\int_{T} \phi_{t}\left(x_{t}\right) d \mu(t)\right) .
\end{aligned}
$$


Example 2 Let $f(t)=g(t)=t^{4}, \Phi_{k}\left(\left(a_{i j}\right)_{1 \leq i, j \leq 3}\right)=\frac{1}{2}\left(a_{i j}\right)_{1 \leq i, j \leq 2}$ and $\mu(\{k\})=1, k=1,2$.

$$
\text { If } X_{1}=\left(\begin{array}{lll}
4 & 1 & 1 \\
1 & 2 & 0 \\
1 & 0 & 1
\end{array}\right) \quad \text { and } \quad X_{2}=\left(\begin{array}{ccc}
5 & -1 & -1 \\
-1 & 2 & 1 \\
-1 & 1 & 3
\end{array}\right), \quad \text { then } X=\left(\begin{array}{cc}
4.5 & 0 \\
0 & 2
\end{array}\right)
$$

and $m_{1}=0.623, M_{1}=4.651, m_{2}=1.345, M_{2}=5.866, m=0.623, M=5.866$ (rounded to three decimal places). We have

$$
\begin{aligned}
\Phi_{1}\left(X_{1}^{4}\right)+\Phi_{2}\left(X_{2}^{4}\right) & =\left(\begin{array}{cc}
629.5 & -87.5 \\
-87.5 & 99
\end{array}\right) \\
<\alpha_{1}\left(\Phi_{1}\left(X_{1}\right)+\Phi_{2}\left(X_{2}\right)\right)^{4}-\delta_{f} \tilde{x} & =\left(\begin{array}{cc}
7823.449 & -53.737 \\
-53.737 & 139.768
\end{array}\right) \\
<\alpha_{1}\left(\Phi_{1}\left(X_{1}\right)+\Phi_{2}\left(X_{2}\right)\right)^{4} & =\left(\begin{array}{cc}
7974.38 & 0 \\
0 & 311.148
\end{array}\right)
\end{aligned}
$$

since $\alpha_{1}=\max _{m_{x} \leq z \leq M_{x}}\left\{\frac{k_{f} z+l_{f}}{g(z)}\right\}=19.447, \delta_{f}=962.73, \widetilde{x}=\left(\begin{array}{cc}0.157 & 0.056 \\ 0.056 & 0.178\end{array}\right)$. Further,

$$
\begin{aligned}
\Phi_{1}\left(X_{1}^{4}\right)+\Phi_{2}\left(X_{2}^{4}\right) & =\left(\begin{array}{cc}
629.5 & -87.5 \\
-87.5 & 99
\end{array}\right) \\
<\alpha_{2}\left(\Phi_{1}\left(X_{1}\right)+\Phi_{2}\left(X_{2}\right)\right)^{4} & =\left(\begin{array}{cc}
5246.13 & 0 \\
0 & 204.696
\end{array}\right) \\
<\alpha_{1}\left(\Phi_{1}\left(X_{1}\right)+\Phi_{2}\left(X_{2}\right)\right)^{4} & =\left(\begin{array}{cc}
7974.38 & 0 \\
0 & 311.148
\end{array}\right),
\end{aligned}
$$

since $\alpha_{2}=\max _{m_{x} \leq z \leq M_{x}}\left\{\frac{k_{f} z+l_{f}-\delta_{f} m_{\tilde{x}}}{g(z)}\right\}=12.794$. We remark that there is no relation between matrices in the right-hand sides of equalities (30) and (31).

Remark 3 Similar to [29, Corollary 4.2], we can determine the constant in the RHS of (29).

(i) Let $f$ be convex. We can determine the value $C$ in

$$
\int_{T} \phi_{t}\left(f\left(x_{t}\right)\right) d \mu(t) \leq C g\left(\int_{T} \phi_{t}\left(x_{t}\right) d \mu(t)\right)
$$

as follows:

- if $g$ is convex, then

$$
C_{\alpha}= \begin{cases}\frac{k_{f} m_{x}+l_{l}-\delta_{f} m_{\tilde{x}}}{g\left(m_{x}\right)} & \text { if } g_{-}^{\prime}(z) \geq \frac{k_{f} g(z)}{k_{f} z+l_{f}-\delta_{f} m_{\tilde{x}}} \text { for every } z \in\left(m_{x}, M_{x}\right), \\ \frac{k_{f} z_{0}+l_{f}-\delta_{f} m_{\tilde{x}}}{g\left(z_{0}\right)} & \text { if } g_{-}^{\prime}\left(z_{0}\right) \leq \frac{k_{f} g\left(z_{0}\right)}{k_{f} z_{0}+l_{f}-\delta_{f} m_{\tilde{x}}} \leq g_{+}^{\prime}\left(z_{0}\right) \\ \frac{k_{f} M_{x}+l_{f}-\delta_{f} m_{\tilde{x}}}{g\left(M_{x}\right)} & \text { for some } z_{0} \in\left(m_{x}, M_{x}\right), \\ \text { if } g_{+}^{\prime}(z) \leq \frac{k_{f} g(z)}{k_{f} z+l_{f}-\delta_{f} m_{\widetilde{x}}} \text { for every } z \in\left(m_{x}, M_{x}\right) ;\end{cases}
$$

- if $g$ is concave, then

$$
C=\max \left\{\frac{k_{f} m_{x}+l_{f}-\delta_{f} m_{\widetilde{x}}}{g\left(m_{x}\right)}, \frac{k_{f} M_{x}+l_{f}-\delta_{f} m_{\tilde{x}}}{g\left(M_{x}\right)}\right\} .
$$


Also, we can determine the constant $D$ in

$$
\int_{T} \phi_{t}\left(f\left(x_{t}\right)\right) d \mu(t) \leq D g\left(\int_{T} \phi_{t}\left(x_{t}\right) d \mu(t)\right)-\delta_{f} \widetilde{x}
$$

in the same way as the above constant $C$ but without $m_{\tilde{x}}$.

(ii) Let $f$ be concave. We can determine the value $c$ in

$$
c g\left(\int_{T} \phi_{t}\left(x_{t}\right) d \mu(t)\right) \leq \int_{T} \phi_{t}\left(f\left(x_{t}\right)\right) d \mu(t)
$$

as follows:

- if $g$ is convex, then $c$ is equal to the right-hand side in (33) with min instead of max;

- if $g$ is concave, then $c$ is equal to the right-hand side in (32) with reverse inequality signs.

Also, we can determine the constant $d$ in

$$
d g\left(\int_{T} \phi_{t}\left(x_{t}\right) d \mu(t)\right)-\delta_{f} \tilde{x} \leq \int_{T} \phi_{t}\left(f\left(x_{t}\right)\right) d \mu(t)
$$

in the same way as the above constant $c$ but without $m_{\widetilde{x}}$.

Theorem 10 and Remark 3 applied to functions $f(z)=z^{p}$ and $g(z)=z^{q}$ give the following corollary, which is a refinement of $[29$, Corollary 4.4$]$.

Corollary 11 Let $\left(x_{t}\right)_{t \in T}$ be a field of strictly positive operators, let $m_{x}$ and $M_{x}, m_{x} \leq M_{x}$, be the bounds of the operator $x=\int_{T} \phi_{t}\left(x_{t}\right) d \mu(t)$. Let $\widetilde{x}$ be defined by (8), $m_{\tilde{x}}$ be the lower bound of the operator $\tilde{x}$ and $\delta_{p}:=m^{p}+M^{p}-2^{1-p}(m+M)^{p}$.

(i) Let $p \in(-\infty, 0] \cup[1, \infty)$. Then

$$
\int_{T} \phi_{t}\left(x_{t}^{p}\right) d \mu(t) \leq C^{\star}\left(\int_{T} \phi_{t}\left(x_{t}\right) d \mu(t)\right)^{q}
$$

where the constant $C^{\star}$ is determined as follows:

- if $q \in(-\infty, 0] \cup[1, \infty)$, then

$$
C^{\star}= \begin{cases}\frac{k_{t} p m_{x}+l_{t} p-\delta_{p} m_{\tilde{x}}}{m_{x}^{q}} & \text { if } \frac{q}{1-q} \frac{l_{t} p-\delta_{p} m_{\tilde{x}}}{k_{t} p} \leq m_{x}, \\ \frac{l_{t} p-\delta_{p} m_{\tilde{x}}}{1-q}\left(\frac{1-q}{q} \frac{k_{t} p}{l_{t} p-\delta_{p} m_{\tilde{x}}}\right)^{q} & \text { if } m_{x} \leq \frac{q}{1-q} \frac{l_{t} p-\delta_{p} m_{\tilde{x}}}{k_{t} p} \leq M_{x}, \\ \frac{k_{t} p M_{x}+l_{t} p-\delta_{p} m_{\tilde{x}}}{M_{x}^{q}} & \text { if } \frac{q}{1-q} \frac{l_{t} p-\delta_{p} m_{\tilde{x}}}{k_{t} p} \geq M_{x}\end{cases}
$$

- if $q \in(0,1)$, then

$$
C^{\star}=\max \left\{\frac{k_{t} p m_{x}+l_{t} p-\delta_{p} m_{\tilde{x}}}{m_{x}^{q}}, \frac{k_{t} p q, M_{x}+l_{t}-\delta_{p} m_{\tilde{x}}}{M_{x}^{q}}\right\} .
$$

Also,

$$
\int_{T} \phi_{t}\left(x_{t}^{p}\right) d \mu(t) \leq D^{\star}\left(\int_{T} \phi_{t}\left(x_{t}\right) d \mu(t)\right)^{q}-\delta_{p} \tilde{x}
$$


holds, where $D^{\star}$ is determined in the same way as the above constant $C^{\star}$ but without $m_{\tilde{x}}$.

(ii) Let $p \in(0,1)$. Then

$$
c^{\star}\left(\int_{T} \phi_{t}\left(x_{t}\right) d \mu(t)\right)^{q} \leq \int_{T} \phi_{t}\left(x_{t}^{p}\right) d \mu(t),
$$

where the constant $c^{\star}$ is determined as follows:

- if $q \in(-\infty, 0] \cup[1, \infty)$, then $c^{\star}$ is equal to the right-hand side in (35) with min instead of $\max$;

- if $q \in(0,1)$, then $c_{\alpha}^{\star}$ is equal to the right-hand side in (34).

Also,

$$
d^{\star}\left(\int_{T} \phi_{t}\left(x_{t}\right) d \mu(t)\right)^{q}-\delta_{p} \tilde{x} \leq \int_{T} \phi_{t}\left(x_{t}^{p}\right) d \mu(t)
$$

holds, where $\delta_{p} \leq 0, \tilde{x} \geq 0$ and $d^{\star}$ is determined in the same way as the above constant $d^{\star}$ but without $m_{\tilde{x}}$.

Using Theorem 10 and Remark 3 for $g \equiv f$ and utilizing elementary calculations, we obtain the following converse of Jensen's operator inequality.

Theorem 12 Let $m_{x}$ and $M_{x}, m_{x} \leq M_{x}$, be the bounds of the operator $x=\int_{T} \phi_{t}\left(x_{t}\right) d \mu(t)$. Iff $:[m, M] \rightarrow \mathbb{R}$ is a continuous convex function and strictly positive on $\left[m_{x}, M_{x}\right]$, then

$$
\int_{T} \phi_{t}\left(f\left(x_{t}\right)\right) d \mu(t) \leq \max _{m_{x} \leq z \leq M_{x}}\left\{\frac{k_{f} z+l_{f}-\delta_{f} m_{\widetilde{x}}}{f(z)}\right\} f\left(\int_{T} \phi_{t}\left(x_{t}\right) d \mu(t)\right)
$$

and

$$
\int_{T} \phi_{t}\left(f\left(x_{t}\right)\right) d \mu(t) \leq \max _{m_{x} \leq z \leq M_{x}}\left\{\frac{k_{f} z+l_{f}}{f(z)}\right\} f\left(\int_{T} \phi_{t}\left(x_{t}\right) d \mu(t)\right)-\delta_{f} \tilde{x}
$$

where $\tilde{x}$ and $\delta_{f}$ are defined by (8) and (9), respectively, and $m_{\widetilde{x}}$ is the lower bound of the operator $\tilde{x}$.

In the dual case, iff is concave, then the reverse inequalities are valid in (36) and (37) with min instead of max.

Furthermore, iff is convex differentiable on $\left[m_{x}, M_{x}\right]$, we can determine the constant

$$
\alpha_{1} \equiv \alpha_{1}\left(m, M, m_{x}, M_{x}, f\right)=\max _{m_{x} \leq z \leq M_{x}}\left\{\frac{k_{f} z+l_{f}-\delta_{f} m_{\tilde{x}}}{f(z)}\right\}
$$

in (36) as follows:

$$
\alpha_{1}= \begin{cases}\frac{k_{f} m_{x}+l_{f}-\delta_{f} m_{\tilde{x}}}{f\left(m_{x}\right)} & \text { if } f^{\prime}(z) \geq \frac{k_{f} f(z)}{k_{f} z+l_{f}-\delta_{f} m_{\tilde{x}}} \text { for every } z \in\left(m_{x}, M_{x}\right), \\ \frac{k_{f} z_{0}+l_{f}-\delta_{f} m_{\tilde{x}}}{f\left(z_{0}\right)} & \text { if } f^{\prime}\left(z_{0}\right)=\frac{k_{f} f\left(z_{0}\right)}{k_{f} z_{0}+l_{f}-\delta_{f} m_{\tilde{x}}} \text { for some } z_{0} \in\left(m_{x}, M_{x}\right), \\ \frac{k_{f} M_{x}+l_{f}-\delta_{f} m_{\tilde{x}}}{f\left(M_{x}\right)} & \text { if } f^{\prime}(z) \leq \frac{k_{f} f(z)}{k_{f} z+l_{f}-\delta_{f} m_{\tilde{x}}} \text { for every } z \in\left(m_{x}, M_{x}\right) .\end{cases}
$$


Also, if $f$ is strictly convex twice differentiable on $\left[m_{x}, M_{x}\right]$, then we can determine the constant

$$
\alpha_{2} \equiv \alpha_{2}\left(m, M, m_{x}, M_{x}, f\right)=\max _{m_{x} \leq z \leq M_{x}}\left\{\frac{k_{f} z+l_{f}}{f(z)}\right\}
$$

in (37) as follows:

$$
\alpha_{2}=\frac{k_{f} z_{0}+l_{f}}{f\left(z_{0}\right)},
$$

where $z_{0} \in\left(m_{x}, M_{x}\right)$ is defined as the unique solution of the equation $k_{f} f(z)=\left(k_{f} z+l_{f}\right) f^{\prime}(z)$ provided $\left(k_{f} m_{x}+l_{f}\right) f^{\prime}\left(m_{x}\right) / f\left(m_{x}\right) \leq k_{f} \leq\left(k_{f} M_{x}+l_{f}\right) f^{\prime}\left(M_{x}\right) / f\left(M_{x}\right)$. Otherwise, $z_{0}$ is defined as $m_{x}$ or $M_{x}$ provided $k_{f} \leq\left(k_{f} m_{x}+l_{f}\right) f^{\prime}\left(m_{x}\right) / f\left(m_{x}\right)$ or $k_{f} \geq\left(k_{f} M_{x}+l_{f}\right) f^{\prime}\left(M_{x}\right) / f\left(M_{x}\right)$, respectively.

In the dual case, iff is concave differentiable, then the value $\alpha_{1}$ is equal to the right-hand side in (38) with reverse inequality signs. Also, iff is strictly concave twice differentiable, then we can determine the value $\alpha_{2}$ in (39) with $z_{0}$, which equals the right-hand side in (39) with reverse inequality signs.

Remark 4 If $f$ is convex and strictly negative on $\left[m_{x}, M_{x}\right]$, then (36) and (37) are valid with min instead of max. If $f$ is concave and strictly negative, then reverse inequalities are valid in (36) and (37).

Applying Theorem 12 to $f(t)=t^{p}$, we obtain the following refinement of [29, Corollary 4.8].

Corollary 13 Let $\left(x_{t}\right)_{t \in T}$ be a field of strictly positive operators, let $m_{x}$ and $M_{x}, m_{x} \leq M_{x}$, be the bounds of the operator $x=\int_{T} \phi_{t}\left(x_{t}\right) d \mu(t)$. Let $\tilde{x}$ be defined by (8), $m_{\widetilde{x}}$ be the lower bound of the operator $\tilde{x}$ and $\delta_{p}:=m^{p}+M^{p}-2^{1-p}(m+M)^{p}$.

If $p \notin(0,1)$, then

$$
\begin{aligned}
0 & \leq \int_{T} \phi_{t}\left(x_{t}^{p}\right) d \mu(t) \leq \bar{K}\left(m_{x}, M_{x}, m, M, p, 0\right)\left(\int_{T} \phi_{t}\left(x_{t}\right) d \mu(t)\right)^{p}-\delta_{p} \\
& \leq \bar{K}\left(m_{x}, M_{x}, m, M, p, 0\right)\left(\int_{T} \phi_{t}\left(x_{t}\right) d \mu(t)\right)^{p} \\
& \leq K(m, M, p)\left(\int_{T} \phi_{t}\left(x_{t}\right) d \mu(t)\right)^{p}
\end{aligned}
$$

and

$$
\begin{aligned}
0 & \leq \int_{T} \phi_{t}\left(x_{t}^{p}\right) d \mu(t) \leq \bar{K}\left(m_{x}, M_{x}, m, M, p, m_{\widetilde{x}}\right)\left(\int_{T} \phi_{t}\left(x_{t}\right) d \mu(t)\right)^{p} \\
& \leq \bar{K}\left(m_{x}, M_{x}, m, M, p, 0\right)\left(\int_{T} \phi_{t}\left(x_{t}\right) d \mu(t)\right)^{p} \\
& \leq K(m, M, p)\left(\int_{T} \phi_{t}\left(x_{t}\right) d \mu(t)\right)^{p}
\end{aligned}
$$


where

$$
\bar{K}\left(m_{x}, M_{x}, m, M, p, c\right)= \begin{cases}\frac{k_{t} p m_{x}+l_{t^{p}}-c \delta_{p}}{m_{x}^{p}} & \text { if } \frac{p\left(l_{t^{p}}-c \delta_{p}\right)}{m_{x}} \geq(1-p) k_{t^{p}}, \\ K(m, M, p, c) & \text { if } \frac{p\left(l_{t^{p}}-c \delta_{p}\right)}{m_{x}}<(1-p) k_{t^{p}}<\frac{p\left(l_{t} p-c \delta_{p}\right)}{M_{x}}, \\ \frac{k_{t} p M_{x}+l_{t p}-c \delta_{p}}{M_{x}^{p}} & \text { if } \frac{p\left(l_{t^{p}}-c \delta_{p}\right)}{M_{x}} \leq(1-p) k_{t^{p}} .\end{cases}
$$

$K(m, M, p, c)$ is a generalization of the known Kantorovich constant $K(m, M, p) \equiv K(m$, $M, p, 0)$ (defined in [6, §2.7]) as follows:

$$
\begin{aligned}
& K(m, M, p, c) \\
& \quad:=\frac{m M^{p}-M m^{p}+c \delta_{p}(M-m)}{(p-1)(M-m)}\left(\frac{p-1}{p} \frac{M^{p}-m^{p}}{m M^{p}-M m^{p}+c \delta_{p}(M-m)}\right)^{p},
\end{aligned}
$$

for $p \in \mathbb{R}$ and $0 \leq c \leq 0.5$.

If $p \in(0,1)$, then

$$
\begin{aligned}
\int_{T} \phi_{t}\left(x_{t}^{p}\right) d \mu(t) & \geq \bar{k}\left(m_{x}, M_{x}, m, M, p, 0\right)\left(\int_{T} \phi_{t}\left(x_{t}\right) d \mu(t)\right)^{p}-\delta_{p} \tilde{x} \\
& \geq \bar{k}\left(m_{x}, M_{x}, m, M, p, 0\right)\left(\int_{T} \phi_{t}\left(x_{t}\right) d \mu(t)\right)^{p} \\
& \geq K(m, M, p)\left(\int_{T} \phi_{t}\left(x_{t}\right) d \mu(t)\right)^{p} \geq 0
\end{aligned}
$$

and

$$
\begin{aligned}
\int_{T} \phi_{t}\left(x_{t}^{p}\right) d \mu(t) & \geq \bar{k}\left(m_{x}, M_{x}, m, M, p, m_{\tilde{x}}\right)\left(\int_{T} \phi_{t}\left(x_{t}\right) d \mu(t)\right)^{p} \\
& \geq \bar{k}\left(m_{x}, M_{x}, m, M, p, 0\right)\left(\int_{T} \phi_{t}\left(x_{t}\right) d \mu(t)\right)^{p} \\
& \geq K(m, M, p)\left(\int_{T} \phi_{t}\left(x_{t}\right) d \mu(t)\right)^{p} \geq 0,
\end{aligned}
$$

where $\bar{k}\left(m_{x}, M_{x}, m, M, p, c\right)$ equals the right-hand side in (42) with reverse inequality signs.

Proof The second inequalities in (40) and (41) follow directly from (37) and (36) by using (39) and (38), respectively. The last inequality in (40) follows from

$$
\begin{aligned}
\bar{K}\left(m_{x}, M_{x}, m, M, p, 0\right) & =\max _{m_{x} \leq z \leq M_{x}}\left\{\frac{k_{t^{p}} z+l_{t^{p}}}{z^{p}}\right\} \\
& \leq \max _{m \leq z \leq M}\left\{\frac{k_{t p} z+l_{t^{p}}}{z^{p}}\right\}=K(m, M, p) .
\end{aligned}
$$

The third inequality in (41) follows from

$$
\bar{K}\left(m_{x}, M_{x}, m, M, p, m_{\widetilde{x}}\right)=\max _{m_{x} \leq z \leq M_{x}}\left\{\frac{k_{t} z+l_{t^{p}}-\delta_{p} m_{\tilde{x}}}{z^{p}}\right\} \leq \bar{K}\left(m_{x}, M_{x}, m, M, p, 0\right),
$$

since $\delta_{p} m_{\widetilde{x}} \geq 0$ for $p \notin(0,1)$ and $M_{x} \geq m_{x} \geq 0$. 


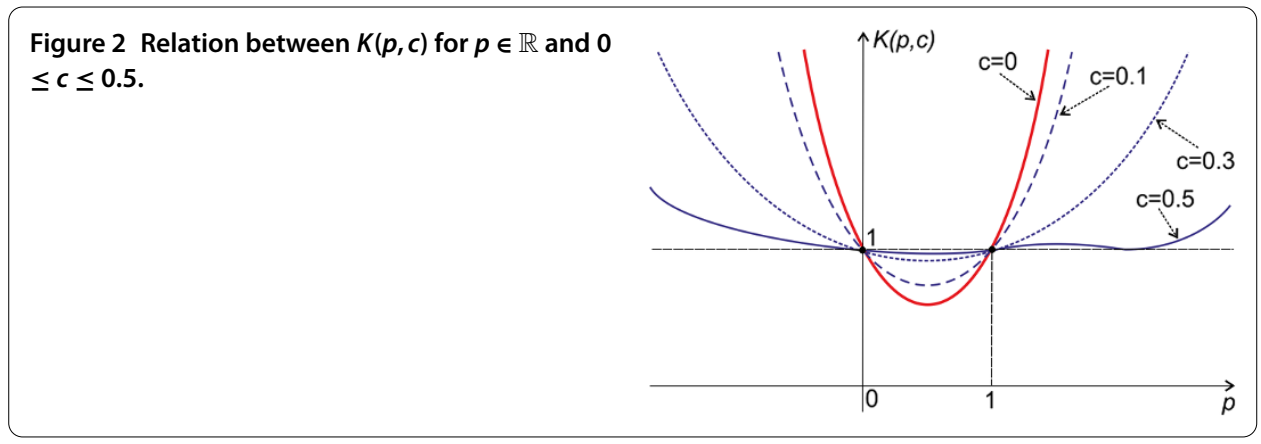

Appendix A: A new generalization of the Kantorovich constant

Definition 1 Let $h>0$. Further generalization of Kantorovich constant $K(h, p)$ (given in [6, Definition 2.2]) is defined by

$$
\begin{aligned}
K(h, p, c):= & \frac{h^{p}-h+c\left(h^{p}+1-2^{1-p}(h+1)^{p}\right)(h-1)}{(p-1)(h-1)} \\
& \times\left(\frac{p-1}{p} \frac{h^{p}-1}{h^{p}-h+c\left(h^{p}+1-2^{1-p}(h+1)^{p}\right)(h-1)}\right)^{p}
\end{aligned}
$$

for any real number $p \in \mathbb{R}$ and any c, $0 \leq c \leq 0.5$. The constant $K(h, p, c)$ is sometimes denoted by $K(p, c)$ briefly. Some of those constants are depicted in Figure 2.

By inserting $c=0$ in $K(h, p, c)$, we obtain the Kantorovich constant $K(h, p)$. The constant $K(m, M, p, c)$ defined by $(43)$ coincides with $K(h, p, c)$ by putting $h=M / m>1$.

Lemma 14 Let $h>0$. The generalized Kantorovich constant $K(h, p, c)$ has the following properties:

(i) $K(h, p, c)=K\left(\frac{1}{h}, p, c\right)$ for all $p \in \mathbb{R}$,

(ii) $K(h, 0, c)=K(h, 1, c)=1$ for all $0 \leq c \leq 0.5$ and $K(1, p, c)=1$ for all $p \in \mathbb{R}$,

(iii) $K(h, p, c)$ is decreasing of $c$ for $p \notin(0,1)$ and increasing of $c$ for $p \in(0,1)$,

(iv) $K(h, p, c) \geq 1$ for all $p \notin(0,1)$ and $0<K(h, 0.5,0) \leq K(h, p, c) \leq 1$ for all $p \in(0,1)$,

(v) $K(h, p, c) \leq h^{p-1}$ for all $p \geq 1$.

Proof (i) We use an easy calculation:

$$
\begin{aligned}
K\left(\frac{1}{h}, p, c\right)= & \frac{h^{-p}-h^{-1}+c\left(h^{-p}+1-2^{1-p}\left(h^{-1}+1\right)^{p}\right)\left(h^{-1}-1\right)}{(p-1)\left(h^{-1}-1\right)} \\
& \times\left(\frac{p-1}{p} \frac{h^{-p}-1}{h^{-p}-h^{-1}+c\left(h^{-p}+1-2^{1-p}\left(h^{-1}+1\right)^{p}\right)\left(h^{-1}-1\right)}\right)^{p} \\
= & \frac{h-h^{p}+c\left(1+h^{p}-2^{1-p}(h+1)^{p}\right)(1-h)}{(p-1)(1-h)} \\
& \times\left(\frac{p-1}{p} \frac{1-h^{p}}{h-h^{p}+c\left(1+h^{p}-2^{1-p}(h+1)^{p}\right)(1-h)}\right)^{p} \\
= & K(h, p, c) .
\end{aligned}
$$

(ii) Let $h>1$. The logarithms calculation and l'Hospital's theorem give $K(h, p, b) \rightarrow 1$ as $p \rightarrow 1, K(h, p, b) \rightarrow 1$ as $p \rightarrow 0$ and $K(h, p, b) \rightarrow 1$ as $h \rightarrow 1+$. Now using (i) we obtain (ii). 
(iii) Let $h>0$ and $0 \leq c \leq 0.5$.

$$
\begin{aligned}
\frac{\mathrm{d} K(h, p, c)}{\mathrm{d} c}= & 2\left(\left(\frac{h+1}{2}\right)^{p}-\frac{h^{p}+1}{2}\right) \\
& \times\left(\frac{p-1}{p} \frac{h^{p}-1}{h-h^{p}+c\left(h^{p}+1-2^{1-p}(h+1)^{p}\right)(h-1)}\right)^{p} .
\end{aligned}
$$

Since the function $z \rightarrow z^{p}$ is convex (resp. concave) on $(0, \infty)$ if $p \notin(0,1)$ (resp. $p \in(0,1)$ ), then $\left(\frac{h+1}{2}\right)^{p} \leq \frac{h^{p}+1}{2}$ (resp. $\left(\frac{h+1}{2}\right)^{p} \geq \frac{h^{p}+1}{2}$ ) for every $h>0$. Then $\frac{\mathrm{d} K(h, p, c)}{\mathrm{d} c} \leq 0$ if $p \notin(0,1)$ and $\frac{\mathrm{d} K(h, p, c)}{\mathrm{d} c} \geq 0$ if $p \in(0,1)$, which gives that $K(h, p, c)$ is decreasing of $c$ if $p \notin(0,1)$ and increasing of $c$ if $p \in(0,1)$.

(iv) Let $h>1$ and $0 \leq c \leq 0.5$. If $p>1$ then

$$
\begin{aligned}
0 & <\frac{(p-1)(h-1)}{h^{p}-h+c\left(h^{p}+1-2^{1-p}(h+1)^{p}\right)(h-1)} \\
& \leq \frac{p-1}{p} \frac{h^{p}-1}{h^{p}-h+c\left(h^{p}+1-2^{1-p}(h+1)^{p}\right)(h-1)}
\end{aligned}
$$

implies

$$
\begin{aligned}
& \frac{(p-1)(h-1)}{h^{p}-h+c\left(h^{p}+1-2^{1-p}(h+1)^{p}\right)(h-1)} \\
& \leq\left(\frac{p-1}{p} \frac{h^{p}-1}{h^{p}-h+c\left(h^{p}+1-2^{1-p}(h+1)^{p}\right)(h-1)}\right)^{p},
\end{aligned}
$$

which gives $K(h, p, c) \geq 1$. Similarly, $K(h, p, c) \geq 1$ if $p<0$ and $K(h, p, c) \leq 1$ if $p \in(0,1)$. Next, using (iii) and [6, Theorem 2.54(iv)], $K(h, p, c) \geq K(h, p, 0) \geq K(h, 0.5,0)$ for $p \in$ $(0,1)$.

(v) Let $p \geq 1$. Using (iii) and [6, Theorem 2.54(vi)], $K(h, p, c) \leq K(h, p, 0) \leq h^{p-1}$.

\section{Competing interests}

The authors declare that they have no competing interests.

\section{Authors' contributions}

All authors contributed equally and significantly in writing this paper. All authors read and approved the final manuscript.

\section{Author details}

'Faculty of Mechanical Engineering and Naval Architecture, University of Zagreb, Ivana Lučića 5, Zagreb, 10000, Croatia. ${ }^{2}$ Faculty of Textile Technology, University of Zagreb, Prilaz baruna Filipovića 30, Zagreb, 10000, Croatia. ${ }^{3}$ Faculty of

Science, Department of Mathematics, University of Split, Teslina 12, Split, 21000, Croatia.

Received: 27 November 2012 Accepted: 9 July 2013 Published: 29 July 2013

\section{References}

1. Davis, C: A Schwarz inequality for convex operator functions. Proc. Am. Math. Soc. 8, 42-44 (1957)

2. Choi, MD: A Schwarz inequality for positive linear maps on $C^{*}$-algebras. III. J. Math. 18, 565-574 (1974)

3. Hansen, F, Pedersen, GK: Jensen's inequality for operators and Löwner's theorem. Math. Ann. 258, 229-241 (1982)

4. Hansen, F, Pedersen, GK: Jensen's operator inequality. Bull. Lond. Math. Soc. 35, 553-564 (2003)

5. Mond, B, Pečarić, J: On Jensen's inequality for operator convex functions. Houst. J. Math. 21, 739-754 (1995)

6. Furuta, T, Mićić Hot, J, Pečarić, J, Seo, Y: Mond-Pečarić Method in Operator Inequalities. Monographs in Inequalities, vol. 1. Element, Zagreb (2005)

7. Hansen, F, Pečarić, J, Perić, I: Jensen's operator inequality and its converses. Math. Scand. 100, 61-73 (2007)

8. Abramovich, S, Jameson, G, Sinnamon, G: Refining Jensen's inequality. Bull. Math. Soc. Sci. Math. Roum. 47, 3-14 (2004)

9. Dragomir, SS: A new refinement of Jensen's inequality in linear spaces with applications. Math. Comput. Model. 52, 1497-1505 (2010) 
10. Fujii, Jl: An external version of the Jensen operator inequality. Sci. Math. Japon. Online 2011, 59-62 (2011)

11. Fujii, JI, Pečarić, J, Seo, Y: The Jensen inequality in an external formula. J. Math. Inequal. 6, 473-480 (2012)

12. Ivelić, A, Matković, A, Pečarić, JE: On a Jensen-Mercer operator inequality. Banach J. Math. Anal. 5, 19-28 (2011)

13. Khosravi, M, Aujla, JS, Dragomir, SS, Moslehian, MS: Refinements of Choi-Davis-Jensen's inequality. Bull. Math. Anal. Appl. 3, 127-133 (2011)

14. Mićić, J, Pavić, Z, Pečarić, J: Extension of Jensen's operator inequality for operators without operator convexity. Abstr. Appl. Anal. 2011, 1-14 (2011)

15. Mićić, J, Pečarić, J, Perić, J: Extension of the refined Jensen's operator inequality with condition on spectra. Ann. Funct. Anal. 3, 67-85 (2012)

16. Moslehian, MS, Kian, M: Jensen type inequalities for Q-class functions. Bull. Aust. Math. Soc. 85, 128-142 (2011)

17. Rooin, J: A refinement of Jensen's inequality. J. Inequal. Pure Appl. Math. 6(2), 38 (2005)

18. Srivastava, HM, Xia, ZG, Zhang, ZH: Some further refinements and extensions of the Hermite-Hadamard and Jensen inequalities in several variables. Math. Comput. Model. 54, 2709-2717 (2011)

19. Xiao, ZG, Srivastava, HM, Zhang, ZH: Further refinements of the Jensen inequalities based upon samples with repetitions. Math. Comput. Model. 51, 592-600 (2010)

20. Wang, LC, Ma, XF, Liu, LH: A note on some new refinements of Jensen's inequality for convex functions. J. Inequal. Pure Appl. Math. 10(2), 48 (2009)

21. Mićić, J, Pavić, Z, Pečarić, J: Jensen's inequality for operators without operator convexity. Linear Algebra Appl. 434, 1228-1237 (2011)

22. Mićić, J, Pečarić, J, Perić, J: Refined Jensen's operator inequality with condition on spectra. Oper. Matrices 7, 293-308 (2013)

23. Mond, B, Pečarić, JE: Converses of Jensen's inequality for linear maps of operators. An. Univ. Timiş., Ser. Mat.-Inform. 2, 223-228 (1993)

24. Mond, B, Pečarić, J: Converses of Jensen's inequality for several operators. Rev. Anal. Numér. Théor. Approx. 23 179-183 (1994)

25. Furuta, T: Operator inequalities associated with Hölder-McCarthy and Kantorovich inequalities. J. Inequal. Appl. 2 137-148 (1998)

26. Mićić, J, Seo, Y, Takahasi, SE, Tominaga, M: Inequalities of Furuta and Mond-Pečarić. Math. Inequal. Appl. 2, 83-111 (1999)

27. Mićić, J, Pečarić, J, Seo, Y, Tominaga, M: Inequalities of positive linear maps on Hermitian matrices. Math. Inequal. Appl. 3, 559-591 (2000)

28. Mićić, J, Pečarić, J, Seo, Y: Converses of Jensen's operator inequality. Oper. Matrices 4, 385-403 (2010)

29. Mićić, J, Pavić, Z, Pečarić, J: Some better bounds in converses of the Jensen operator inequality. Oper. Matrices 6 , 589-605 (2012)

30. Mitrinović, DS, Pečarić, JE, Fink, AM: Classical and New Inequalities in Analysis. Kluwer Academic, Dordrecht (1993)

doi:10.1186/1029-242X-2013-353

Cite this article as: Mićić et al.: Refined converses of Jensen's inequality for operators. Journal of Inequalities and Applications 2013 2013:353.

\section{Submit your manuscript to a SpringerOpen ${ }^{\ominus}$ journal and benefit from:}

- Convenient online submission

- Rigorous peer review

- Immediate publication on acceptance

Open access: articles freely available online

- High visibility within the field

- Retaining the copyright to your article 